\title{
Are Differences in Dysphagia Assessment, Oral Care Provision, or Nasogastric Tube Insertion Associated with Stroke-Associated Pneumonia? A Nationwide Survey Linked to National Stroke Registry Data
}

\author{
Sabrina A. Eltringham ${ }^{a, b}$ Benjamin D. Bray ${ }^{c}$ Craig J. Smith $^{d, e}$ Sue Pownall ${ }^{a}$ \\ Karen Sage ${ }^{b}$ \\ aSpeech and Language Therapy Department, Sheffield Teaching Hospitals NHS Foundation Trust, Sheffield, UK; \\ ${ }^{b}$ Faculty of Health and Education, Manchester Metropolitan University, Manchester, UK; 'SChool of Population \\ Health and Environmental Sciences, King's College London, London, UK; ${ }^{\mathrm{d} M a n c h e s t e r ~ C e n t r e ~ f o r ~ C l i n i c a l ~}$ \\ Neurosciences, Manchester Academic Health Science Centre, Salford Royal NHS Foundation Trust, Salford, UK; \\ eDivision of Cardiovascular Sciences, School of Medical Sciences, University of Manchester, Manchester, UK
}

\section{Keywords}

Stroke-associated pneumonia - Dysphagia assessment ·

Acute stroke · Nasogastric tube feeding · Oral care

\begin{abstract}
Introduction: Stroke-associated pneumonia (SAP) is a common complication associated with poor outcomes. Early dysphagia screening and specialist assessment is associated with a reduced risk of SAP. Evidence about oral care and nasogastric tube (NGT) placement is equivocal. This study aimed to expose variations in dysphagia management practices and explore their associations with SAP. Participants and Methods: Speech pathologists from 166 stroke units in England and Wales were surveyed about dysphagia assessment and management, oral care, and NGT placement. Survey data were then linked to the Sentinel Stroke National Audit Programme (SSNAP), the national register of stroke. Univariable and multivariable linear regression models were fitted to estimate the association between dysphagia man-
\end{abstract}

agement practices and SAP incidence. Results: 113 hospitals completed the survey (68\%). Variation was evident in dysphagia screening protocols (DSPs), oral care, and NGT practice while specialist swallow assessment data patterns were more consistent. Multivariable analysis showed no evidence of an association in incidence of SAP when using a wateronly hospital DSP compared to a multiconsistency DSP (B $-0.688,95 \% \mathrm{Cl}:-2.912$ to 1.536 ), when using written swallow assessment guidelines compared to not using written guidelines (B $0.671,95 \% \mathrm{Cl}:-1.567$ to 2.908 ), when teams inserted NGTs overnight compared to teams which did not (B $-0.505,95 \% \mathrm{Cl}:-2.759$ to 1.749 ), and when teams had a written oral care protocol compared to those which did not ( $\mathrm{B}-1.339,95 \% \mathrm{Cl}:-3.551$ to 0.873 ). Discussion and Conclusion: Variation exists in dysphagia screening and management, but there was no evidence of an association between clinical practice patterns and incidence of SAP. Further research with larger sample sizes is needed to examine association with SAP.

(c) 2021 The Author(s)

Published by S. Karger AG, Basel karger@karger.com www.karger.com/ced

Karger ${ }^{\prime \prime} \div$

GOPEN ACCESS
(C) 2021 The Author(s)

Published by S. Karger AG, Basel

This is an Open Access article licensed under the Creative Common Attribution-NonCommercial-4.0 International License (CC BY-NC) (http://www.karger.com/Services/OpenAccessLicense), applicable to the online version of the article only. Usage and distribution for commercial purposes requires written permission.
Correspondence to:

Sabrina A. Eltringham, sabrina.eltringham@stu.mmu.ac.uk 


\section{Introduction}

Stroke-associated pneumonia (SAP) is a frequent complication in acute stroke and a significant predictor of mortality [1]. Dysphagia is a main risk factor and occurs in $37-78 \%$ of acute stroke patients [2]. The drive to decrease this risk has resulted in standards of dysphagia care around the world, and screening and specialist swallow assessment is included in the auditing process for hospital stroke units [3].

Early detection of dysphagia is recommended [4]. The UK Guidelines recommend a validated dysphagia screen [5]. In practice, a range of dysphagia screening protocols (DSPs) are used [6]. The clinical swallowing assessment (usually undertaken by speech and language pathologists [SLP]) also shows a high degree of variability [7]. The level of detail about dysphagia management during the first $72 \mathrm{~h}$ of admission after stroke is limited [6].

The aim of this study was to use survey data to gain a greater understanding of dysphagia assessment and management practice and other related clinical processes specifically oral care and NGT feeding during the first 7 days of hospital admission. Our objectives were to reveal variations in practice in hospital stroke units who also participated in a large national audit registry in order to use our survey data alongside audit data to estimate associations with SAP.

\section{Methods}

\section{Study Design and Data Source}

A national, cross-sectional survey of SLP Clinical Leads in Acute Stroke was undertaken. The sample frame used hospital stroke units registered with the Sentinel Stroke National Audit Programme (SSNAP), the national register of stroke in England and Wales. Hospital Teams were included if they were registered during October-December 2019, had sufficient records to report, and were actively admitting stroke patients. One hundred and sixty-six teams were included. SAP was defined as the administration of antibiotics for a new clinical diagnosis of pneumonia in the first 7 days after admission [4]. Data from the survey were linked to SSNAP Hospital Level Patient Centred Post-72-h data. Quarterly data from 2019 were used to create a January-December annual data set.

\section{Development and Pretesting of the Survey}

A tailored survey design underpinned by social exchange theory was used [8]. The topics and question objectives were identified from a series of studies [6, 9-11]. The survey was pretested before fielding the questionnaire to the target population. The definitive version of the survey included 51 questions grouped into 4 topic areas: (a) dysphagia screening, (b) specialist swallowing assessments and management, (c) NGT feeding, and (d) oral care processes.
Recruitment Process and Description of the Sample

The survey was a non-open survey using a hyperlink sent only to the SLP Clinical Lead for Acute Stroke. These people were identified through the research teams' professional networks. The respondent answered the survey on behalf of their stroke unit.

\section{Survey Administration}

The hyperlink to the e-survey was sent through email which opened to the survey web page. Survey responses were captured automatically on the Qualtrics survey platform. The survey was opened on September 2, 2020, for 1 month. The hyperlink remained active after the intended closing date to allow for any late responders due to the second wave of the COVID-19 pandemic. Only completed surveys were included. The CHERRIES checklist [12] was used to ensure complete description of the survey methodology (shown in online suppl. Table 1; for all online suppl. material, see www.karger.com/doi/10.1159/000519903).

\section{Statistical Analysis}

The statistical analysis plan was registered on ClinicalTrials. gov (Identifier: NCT04779710). Completed survey responses were exported to SPSS for Windows (Version 26.0) predictive analytics software for analysis. Descriptive statistics were used for categorical and continuous data. A simple linear regression analysis using a coefficient model and $95 \%$ confidence intervals was used to explore associations between the dependent variable (incidence of SAP) and the independent binary variables.

Sensitivity analysis was undertaken to understand how certain values may contribute to the overall uncertainty of the statistical model. First, the impact of lower-than-average response rates from a small group of regions was explored. Second, the latest SSNAP annual data (April 19/March 20) were used to explore the possible confounding effect of the beginning of the global COVID-19 pandemic. Finally, all 4 factors were grouped together to run a multivariable model to see what the combined effect would be on the independent variables.

\section{Results}

One hundred and thirteen completed surveys were included in the analysis. The completeness rate was $68.1 \%$. The overall incidence of SAP was $9.26 \%$ (SD 5.11). The characteristics of the cohort are shown in Table 1 .

\section{Dysphagia Screening Protocols}

One hundred and eight teams (95.6\%) used a written DSP. In hospital teams who used a written DSP, $97.2 \%$ had mandatory training for the person conducting the screen. Stroke Nurse Specialists (SNSs) and Registered Nurses (RNs) most frequently carried out the screen. Some units described different models of screening which included SNSs trained to Specialist Level on the InterProfessional Dysphagia Framework (IDF) [13] to carry out the specialist swallow assessment on admission; SNSs 
Table 1. Demographic characteristics by region

\begin{tabular}{lllll}
\hline Region & $\begin{array}{l}\text { RAT responses, } \\
\% \text { RR by region }\end{array}$ & $\begin{array}{l}\text { N-RAT responses, } \\
\% \text { RR by region }\end{array}$ & $\begin{array}{l}\text { Total responses, } \\
\% \text { RR by region }\end{array}$ & $\begin{array}{l}\text { Mean (SD) SAP incidence as } \\
\% \text { of all stroke admissions }\end{array}$ \\
\hline London & $5(62.5)$ & $15(75.0)$ & $20(71.4)$ & $10.89(6.8)$ \\
East Midlands & $7(100.0)$ & $0(\mathrm{n} / \mathrm{a})$ & $7(100.0)$ & $6.05(3.3)$ \\
East of England & $11(78.6)$ & $0(\mathrm{n} / \mathrm{a})$ & $11(73.3)$ & $8.74(5.7)$ \\
West Midlands & $9(69.2)$ & $0(\mathrm{n} / \mathrm{a})$ & $9(64.2)$ & $8.75(4.9)$ \\
GM and Cheshire & $1(33.3)$ & $2(33.3)$ & $3(33.3)$ & $8.91(3.5)$ \\
North West Coast & $10(83.3)$ & $1(50)$ & $11(78.6)$ & $8.43(6.0)$ \\
North of England & $6(75.0)$ & $3(75.0)$ & $9(75.0)$ & $11.87(5.4)$ \\
Yorks and Humber & $5(55.6)$ & $4(80.0)$ & $9(64.3)$ & $7.89(3.3)$ \\
South East & $6(54.5)$ & $2(100.0)$ & $8(61.5)$ & $11.10(4.0)$ \\
South West & $14(100.0)$ & $0(\mathrm{n} / \mathrm{a})$ & $14(100.0)$ & $8.68(4.1)$ \\
Thames Valley & $1(25.0)$ & $0(\mathrm{n} / \mathrm{a})$ & $1(25.0)$ & $9.56()$. \\
Wessex & $6(85.7)$ & $0(\mathrm{n} / \mathrm{a})$ & $6(85.7)$ & $10.77(4.5)$ \\
Wales & $5(41.7)$ & $0(0)$ & $5(33.3)$ & $6.04(1.4)$ \\
\hline Total & $86(70.5)$ & $27(61.4)$ & $113(68.1)$ & $9.26(5.1)$ \\
\hline
\end{tabular}

Values are $n(\%)$ unless otherwise stated. RAT, Routinely Admitting Team; N-RAT, Non-routinely Admitting Acute Team*; RR, response rate; SD, standard deviation; SAP, stroke-associated pneumonia. * Non-routinely admitting acute teams are teams which do not generally admit stroke patients directly but continue to provide care in an acute setting when patients have been transferred from their place of initial treatment.

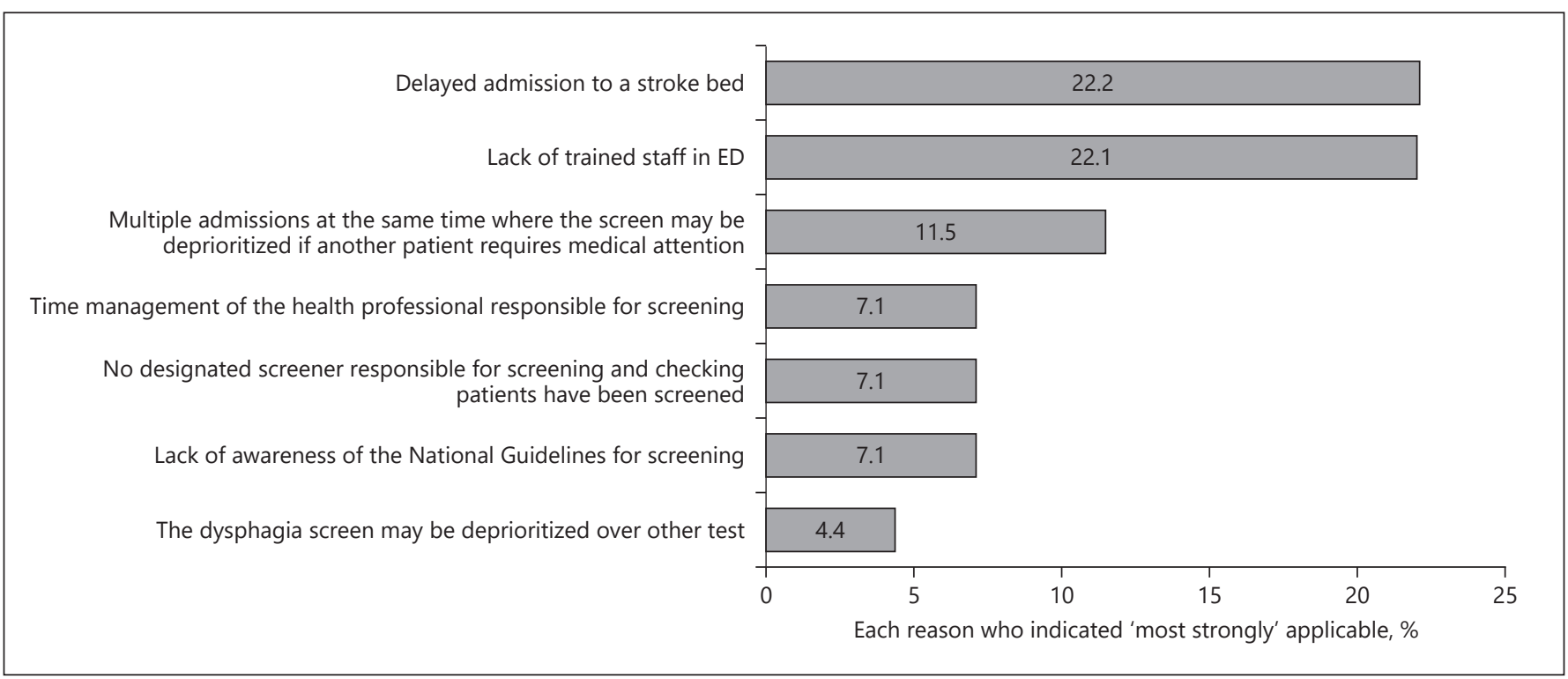

Fig. 1. Reasons for delays in dysphagia screening.

and RNs within the same unit trained to different competency levels to screen with a water and multiple consistency screen; and a 2-tier screening process where a water-only screen was undertaken in Emergency Department, and depending on the outcome, the patient was screened more comprehensively on the Stroke Unit. Oth- er models included patients receiving up to 3 swallow screens in the first $24 \mathrm{~h}$.

Ten teams used published dysphagia screens. Eight different screens were used (shown in online suppl. Fig. 1). One response was excluded due to an incorrect response. One hundred and two hospital teams (90.3\%) used a lo- 


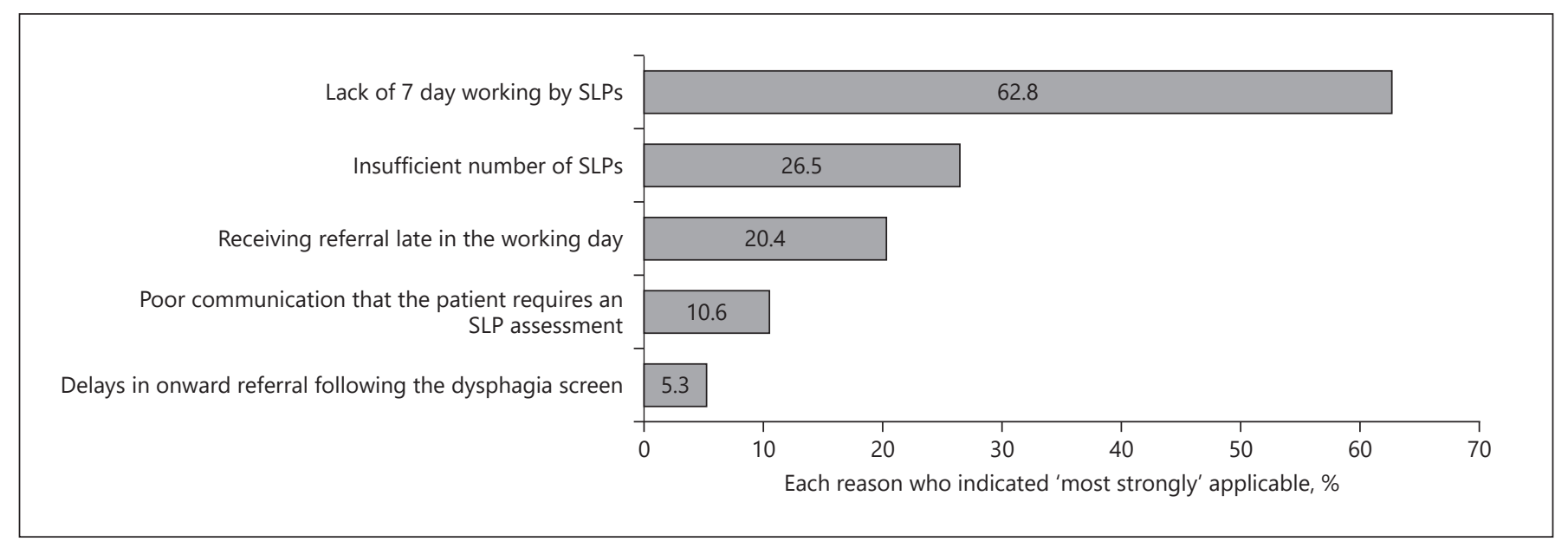

Fig. 2. Reasons for delays in specialist swallow assessment.

cally developed DSP. Ninety-five percent involved either an indirect swallow test or an indirect swallow test and oromotor test. Fifty-three (52\%) of the 102 teams that used a locally developed DSP used a water-only DSP compared to a multiconsistency screen. The maximum amount of water ranged from $10 \mathrm{~mL}$ to $200 \mathrm{~mL}$. In the 49 teams which used a multiconsistency DSP, International Dysphagia Diet Standardisation Initiative (IDDSI) Level 0 Thin Fluids, Level 4 Pureed, and Level 7 Regular Diet were most frequently used [14]. Level 0 was used first by $91.8 \%(N=45)$ of teams compared to $8.2 \%(N=4)$ which began screening oral intake with Level 3 Moderately Thick fluids.

Reasons for delays in patients being screened for dysphagia are shown in Figure 1. All teams referred patients to an appropriately trained professional for a clinical swallow assessment if dysphagia was identified.

\section{Clinical Swallow Assessment}

One team used a published clinical swallow assessment: The Mann Assessment of Swallowing Ability (MASA), and 36 teams (32\%) used other written guidelines; locally developed guidance was mainly used. Seventy-six out of 102 hospital teams (67.3\%) did not use written guidelines. The swallowing assessment involved gathering information about the patient's medical history and presenting condition, assessment of the patient's cognition, communication, and respiratory status, a cranial nerve examination, and assessment with diet and fluids. There was the potential for the full range of the IDDSI levels to be included. Forty-six percent of teams used postural techniques, $35 \%$ used swallowing manoeuvres, and $15 \%$ used cough reflex testing.
Assessment with postural techniques and swallowing manoeuvres was dependent on the patient's physical and cognitive status. Five teams assessed these using videofluroscopy (VFS) rather than at the patient's bedside. Reasons for delays in patients having a clinical swallow assessment are shown in Figure 2.

\section{Instrumental Swallowing Assessments}

One hundred and nine teams (96.5\%) had access to instrumental swallowing assessments. Of those who had access to VFS and fibre optic endoscopic evaluation of swallowing (FEES), 15 of the 52 teams (29\%) would typically use these assessments if clinically indicated within the first 7 days of admission, compared to 9 of the 56 teams $(16.1 \%)$ who had access to VFS only. The 1 team that had FEES only would typically use it within the first 7 days if indicated.

\section{Treatment Options}

Diet and fluid modification (100\%), NGT feeding (98\%), postural techniques (59\%), oro-motor exercises (54\%), swallowing manoeuvres (49\%), pharmacological management $(38 \%)$, sensory stimulation $(33 \%)$, Frazier Free Water Protocol (24\%), biofeedback (7\%), and electrical stimulation (3\%) were options recommended during the first 7 days of a patient's admission.

\section{Nasogastric Tube Feeding}

One hundred and one hospital teams (89.4\%) had a written NGT protocol. Time from decision to non-orally feed and feed by NGT was $43.4 \%(<6 \mathrm{~h}$ ), $32.7 \%$ ( $\geq 6$ to $<12 \mathrm{~h}$ ), $14.2 \%$ ( $\geq 12$ to $<24 \mathrm{~h})$, and $9.7 \%(\geq 24$ to $<48 \mathrm{~h})$. 
Table 2. Coefficient ${ }^{a}$ multivariable analysis

\begin{tabular}{|c|c|c|c|c|c|c|c|}
\hline \multirow[t]{2}{*}{ Model } & \multicolumn{5}{|c|}{ Unstandardized coefficients } & \multicolumn{2}{|c|}{$\begin{array}{l}95.0 \% \text { confidence } \\
\text { interval for B }\end{array}$} \\
\hline & sample & B & std. error & $t$ & sig. & $\begin{array}{l}\text { lower } \\
\text { bound }\end{array}$ & $\begin{array}{l}\text { upper } \\
\text { bound }\end{array}$ \\
\hline \multicolumn{8}{|l|}{ Type of DSP } \\
\hline Water only (reference) versus water and other consistencies & 102 & -0.688 & 1.120 & -0.614 & 0.541 & -2.912 & 1.536 \\
\hline Overnight NGT insertion (reference) versus no overnight NGT insertion & 102 & -0.505 & 1.136 & -0.444 & 0.658 & -2.759 & 1.749 \\
\hline \multicolumn{8}{|l|}{ Written oral care protocol } \\
\hline $\begin{array}{l}\text { Hospitals with a written oral care protocol (reference) versus hospitals } \\
\text { without a written oral care protocol }\end{array}$ & 102 & -1.339 & 1.115 & -1.201 & 0.233 & -3.551 & 0.873 \\
\hline
\end{tabular}

${ }^{\text {a }}$ Dependent variable: antibiotics for newly acquired pneumonia in the first 7 days from clock start using SSNAP 2019 Patient Centred Post-72-h data.

Sixty-four out of 113 hospital teams (56.6\%) inserted NGTs overnight. Confirmation of NGT placement was done by $\mathrm{pH}$ testing of the NGT aspirate (74.3\%) and chest radiography if no aspirate was obtained or $\mathrm{pH}$ was above the recommended level (85.8\%). Nineteen teams (16.8\%) routinely performed chest radiography before starting feeding.

The standard position for feeding was a minimum of $30^{\circ}$, with $40.7 \%$ of teams specifying $45^{\circ}$ as the standard. Mittens (94\%), nasal bridles (83\%), and 1:1 patient staff supervision (64\%) were used in cases of inadvertent NGT removal. Responses varied about the maximum number of times the NGT would be reinserted in any patient.

\section{Oral Care}

Seventy-two $(63.7 \%)$ of the 113 teams had a written oral care protocol. In 11 (15.3\%), the protocol was specifically for stroke patients. Clinical Support Workers and Health Care Assistants, RNs, SLPs, and Nursing Associates or Nursing Apprentices most frequently carried out oral care. In 76 teams (67.3\%), staff received training. In $26(34.2 \%)$, training was specific to the oral care of stroke patients. Training included ward-based $(53.1 \%)$, classroom-based (19.5\%), and online training (17.7\%).

Ninety-five teams (84.1\%) did not consider oral care in acute stroke as any different to the oral care in other parts of the stroke pathway. Seventy-one teams (62.8\%) did perceive there to be differences in oral care needs and provision for patients with dysphagia which included increased frequency of oral care, patient-specific recommendations, and use of specialist mouth care products and equipment. In patients who were NBM, less frequent oral care was justified because patients were drowsy, and staff worried about risk of aspiration. There was a wide variation in the frequency of oral care (every $1-2 \mathrm{~h}$ to once a day). The most frequently used interventions were removal of excess secretions (100\%) and brushing of teeth and cleaning of gums with toothpaste (96\%) compared to chlorhexidine gel (35\%) (shown in online suppl. Fig. 2, 3).

\section{Associations between Described Care Processes and SAP}

The univariable (shown in online suppl. Table 2) and multivariable (shown in Table 2) analyses indicate no evidence of an association in the incidence of SAP when comparing teams who use a water-only hospital DSP compared to teams who use water and other consistencies, nor when comparing teams who use written assessment guidelines for their clinical assessment of swallowing compared to those who did not. Similarly, there was no link between incidence of SAP and whether teams inserted NGTs overnight or teams who did not, nor teams who had a written oral care protocol compared to those who did not.

\section{Sensitivity Analysis}

Findings were unchanged when using the SSNAP April 19-March 20 Patient Centred Post-72-h cohort data and when the low \% respondent regions, GM and Cheshire, Thames Valley, and Wales, were excluded from the model (shown in online suppl. Tables 3,4 ). 


\section{Discussion}

National registers such as SSNAP allow us to record timings of care processes potentially relevant to SAP by recording actions taken in the first $72 \mathrm{~h}$, important because patients are increasingly susceptible to infection in the first days after stroke. Our research aimed to unpick what we know are a multifactorial and complex set of factors, by revealing variations in organizational practice and find out what if any has an impact on the incidence of SAP. Four care process issues that might contribute to SAP were identified (using a DSP that uses 100\% water compared to a DSP that uses water and other consistencies, using written guidelines for the first specialist swallow assessment, insertion of NGTs overnight, and using a written oral care protocol).

We found variation across hospital teams in dysphagia screening, specialist swallow assessment and management, oral care, and NGT care processes during the first 7 days of hospital admission. In this study, clinical equipoise was almost 50:50 when choosing whether to use water-only DSPs or screening protocols which used multiple consistencies. In the absence of comparative studies to determine whether the outcome is better between the different screening regimes, patients with acute stroke should be screened with a formal dysphagia screening test as fast as possible after admission to the hospital using either water or multiple consistency tests [3].

Early screening of dysphagia and specialist swallow assessments are associated with reduced risk of SAP $[4,15$, 16]. Our study identified the most frequently reported reasons for delay. The clinical implication of this finding is that some of the reasons for delay are modifiable and have the potential to contribute to reducing the incidence of SAP. For example, having enough trained staff in Emergency Department to screen patients on admission, improving patient flow to a designated stroke bed, 7-day SLP working, and training SNSs to Specialist Level on the IDF would potentially lead to patients being assessed by a specialist sooner and thereby reduce risk of patients developing SAP.

This survey provided further information about what is included in the clinical swallow assessment and new knowledge about SLP management options. Our study concurred with McAllister et al. [17] that there are core components in the clinical bedside assessment. We also revealed variances. These differences may arise based on individual patient presentation, development and testing of hypotheses [17], organizational protocols (i.e., use of cough reflex testing), and evidence-based practice (i.e., use of instrumental assessments to inform management).
This survey found that less than two-thirds of our sample had a written oral protocol and approximately $90 \%$ had a written NGT protocol. There was variation in oral care and NGT processes. There is a continuing paucity of good-quality evidence about oral care interventions in acute stroke [18], and to date, the evidence that NGT placement increases risk of SAP is equivocal [11]. Randomized control trials such as the CHOSEN feasibility trial [19] which is investigating oral healthcare interventions in stroke patients with dysphagia are needed to inform clinical guidelines.

The results of this study did not find evidence of an association between clinical practice patterns and incidence of SAP. The study was robust in survey design, had a high completion rate, and these data were used in combination with data drawn from an established national registry. There are limitations to the survey design used which may prevent the study from detecting any associations between the variables explored here and SAP. There is a possibility of misclassification error although this was minimized by careful design to ensure that the most appropriate person was surveyed in order that the data were as accurate as possible. Another potential threat to validity is the incidence of SAP reported by the National Stroke Audit for the period of analysis. While this is possible, the incidence of SAP has remained stable since the start of the SSNAP registry in 2013 (mean 8.56, SD 0.28) and is consistent with the wider literature [20]. It is possible that the size of the effect may have been too small to detect, and an effect may have been picked up in a larger group of hospital teams. The authors aimed to minimize other potential limitations, including the possibility of recall bias by employing a tailored survey design and pretesting the survey.

Trying to unpack and identify which factors may contribute to risk of SAP is challenging. Some of the processes such as dysphagia screening and the specialist swallow assessment occur sequentially in a patient's pathway while others (such as implementation of the SLP care plan and oral care) are ubiquitous, underpinning care throughout the whole hospital stay; meanwhile, placement and confirmation of the NGT may be carried out more than once and will happen in a less predictable way based on the patient requirement making it difficult to unpack. Additionally, different professional groups are involved in delivering these care processes. Aoki et al. [21] found that an MDT approach to swallowing reduced incidence of SAP. To understand the difference of this approach, frequencies of professional oral care and instrumental swallowing evaluations before and after team organiza- 
tion were evaluated. Our research identified a potential underuse of instrumental swallowing assessments when clinically indicated during the first 7 days of admission. In a comparative survey of German stroke units [22], FEES was more readily available than VFS, with $71 \%$ of stroke units having access to FEES. This may be in part due to FEES being a criterion for Stroke Unit accreditation [23].

Recommendations for future research might include further exploration of the link between screening methods and SAP and undertake a feasibility trial and randomize patients to 1 of 2 treatment groups: one using a water-only DSP and the second group using water and other consistencies, to assess the relative association of SAP of these 2 screening regimes. Additionally, a larger sample at both registry and survey level might show associations which this national sample was unable to detect. A European-wide stroke registry and survey might provide a large enough sample to detect important variations if the same methodology were used. Further evaluation of care processes such as SLT management practices, for example, use of limited oral trials for those at risk of aspiration if full amounts are taken orally [24], is warranted.

\section{Acknowledgments}

We would like to thank the participants, Sentinel Stroke National Audit Programme (SSNAP), for their contribution as data providers, as well as the people and organizations participating in SSNAP. The research team acknowledges the support of the National Institute for Health Research Clinical Research Network (NIHR CRN).

\section{Statement of Ethics}

This study protocol was reviewed and approved by the London-Bromley Research Ethics Committee (REC Ref 18/LO/0096), and the Manchester Metropolitan University REC (EthOS ID 25470) approved this study. Participation was voluntary. Participants were asked to confirm that they had read the information sheet and told by completing and submitting the survey they were providing consent for the data included to be included in the final analysis.

\section{Conflict of Interest Statement}

The authors have no conflicts of interest to declare.

\section{Funding Sources}

This work was supported by the Stroke Association (Grant No. SE-TSA PGF 2017/03).

\section{Author Contributions}

S.A.E. researched the literature, and S.A.E., B.D.B., C.J.S., S.P., and K.S. conceived the study. S.A.E., B.D.B., C.J.S., S.P., and K.S. were involved in protocol development, S.A.E. and K.S. in gaining ethical approval, S.A.E. in recruitment, and S.A.E. and B.D.B. in data analysis. S.A.E. wrote the first draft of the manuscript. All authors reviewed and edited the manuscript and approved the final version of the manuscript.

\section{Data Availability Statement}

All data generated or analysed during this study are included in this article and its online supplementary material files. Further enquiries can be directed to the corresponding author.

\section{References}

1 Westendorp WF, Nederkoorn PJ, Vermeij JD, Dijkgraaf MG, van de Beek D. Post-stroke infection: a systematic review and meta-analysis. BMC Neurol. 2011;11(1):110.

2 Martino R, Foley N, Bhogal S, Diamant N, Speechley M, Teasell R. Dysphagia after stroke: incidence, diagnosis, and pulmonary complications. Stroke. 2005 Dec;36(12): 2756-63.

3 Dziewas R, Michou E, Trapl-Grundschober M, Avtar L, Arsava E, Bath P, et al. European stroke organization and European society for swallowing disorders guideline for the diagnosis and treatment of post-stroke dysphagia. Eur Stroke J. 2021. Sep;6(3):LXXXIX-CXV

4 Bray BD, Smith CJ, Cloud GC, Enderby P, James M, Paley L, et al. The association between delays in screening for and assessing dysphagia after acute stroke, and the risk of stroke-associated pneumonia. J Neurol Neurosurg Psychiatry. 2017 Jan;88(1):25-30.

5 ISWP. National clinical guideline for stroke. 5th ed. London: Royal College of Physicians; 2016.

6 Eltringham SA, Kilner K, Gee M, Sage K, Bray $\mathrm{BD}$, Pownall S, et al. Impact of dysphagia assessment and management on risk of strokeassociated pneumonia: a systematic review. Cerebrovasc Dis. 2018;46(3-4):99-107.

7 McAllister S, Kruger S, Doeltgen S, Tyler-Boltrek E. Implications of variability in clinical bedside swallowing assessment practices by speech language pathologists. Dysphagia. 2016;31(5):650-62.

8 Dillman DA, Smyth JD, Christian LM. Internet, phone, mail, and mixed mode surveys: the tailored design method. 4th ed. Indianapolis, IN: John Wiley \& Sons Inc; 2014.
9 Eltringham SA, Pownall S, Bray B, Smith CJ, Piercy L, Sage K. Experiences of dysphagia after stroke: an interview study of stroke survivors and their informal caregivers. Geriatrics. 2019 Dec 7;4(4):67.

10 Eltringham SA, Smith CJ, Pownall S, Sage K, Bray B. Variation in dysphagia assessment and management in acute stroke: an interview study. Geriatrics. 2019 Oct 25;4(4):60.

11 Eltringham SA, Kilner K, Gee M, Sage K, Bray BD, Smith CJ, et al. Factors associated with risk of stroke-associated pneumonia in patients with dysphagia: a systematic review. Dysphagia. 2020 Oct;35(5):73544.

12 Eysenbach G. Improving the quality of web surveys: the checklist for reporting results of internet e-surveys (CHERRIES). J Med Internet Res. 2004;6(3):e34.
Are Differences in Dysphagia

Management Associated with Pneumonia?
Cerebrovasc Dis 2022;51:365-372 DOI: $10.1159 / 000519903$ 
13 Boaden L, Davis S. Inter professional dysphagia framework. In: Storey L, Watkins C, editors. National dysphagia competence steering group; 2006.

14 Initiative IDDS. Complete IDDSI framework detailed definitions. 2019. Available from: https: //iddsi.org/IDDSI/media/images/ Complete_IDDS I_Framework Final_31July2019.pdf2019.

15 Al-Khaled M, Matthis C, Binder A, Mudter J, Schattschneider J, Pulkowski U, et al. Dysphagia in patients with acute ischemic stroke: early dysphagia screening may reduce stroke-related pneumonia and improve stroke outcomes. Cerebrovasc Dis. 2016;42(1-2):81-9.

16 Dhufaigh NN, Hayes M. 128 the sooner the better: does early speech and language therapy involvement in stroke management result in better dysphagia outcomes? Age Ageing. 2017;46(3):iii13-59.
17 McAllister S, Tedesco H, Kruger S, Ward EC, Marsh C, Doeltgen SH. Clinical reasoning and hypothesis generation in expert clinical swallowing examinations. Int J Lang Commun Disord. 2020 Jul;55(4):480-92.

18 Campbell P, Bain B, Furlanetto DL, Brady MC. Interventions for improving oral health in people after stroke. Cochrane Data Sys Rev. 2020;2020(12):CD003864.

19 Smith CJ. CHlorhexidine Or toothpaSte, manual or powered brushing to prEvent pNeumonia complicating stroke (CHOSEN): a $2 \times 2$ factorial randomised controlled feasibility trial; 2021. Available from: https://fundingawards.nihr.ac.uk.

20 Hannawi Y, Hannawi B, Rao CP, Suarez JI, Bershad EM. Stroke-associated pneumonia: major advances and obstacles. Cerebrovasc Dis. 2013;35(5):430-43.
21 Aoki S, Hosomi N, Hirayama J, Nakamori M, Yoshikawa M, Nezu T, et al. The multidisciplinary swallowing team approach decreases pneumonia onset in acute stroke patients. PloS One. 2016;11(5): e0154608.

22 Flader CM, Rosendahl C, Günther T. Leitlinienkonforme Dysphagiediagnostik. Nervenarzt. 2017;88(10):1168-76.

23 Dziewas R, Allescher H-D, Aroyo I, Bartolome G, Beilenhoff U, Bohlender J, et al. Diagnosis and treatment of neurogenic dysphagia - S1 guideline of the. German Soc Neurol. 2021;3(1):23

24 Julier R, Benfield JK. Evaluating the use of oral trials for inpatient dysphagia management: an initial cross-sectional database study. Am J Speech Lang Pathol. 2021 Jul 14;30(4):1793804 